\title{
Independent Expression of Fibril-forming Collagens I, II, and III in Chondrocytes of Human Osteoarthritic Cartilage
}

\author{
Thomas Aigner, Wolf Bertling, Hartmut Stöss, ${ }^{*}$ Gerd Weseloh, ${ }^{\star}$ and Klaus von der Mark \\ Departments of *Pathology and ${ }^{\ddagger}$ Orthopaedics, and Max-Planck-Society, Clinical Research Units for Rheumatology \\ at the Medical Clinic III, University of Erlangen-Nürnberg, 8520 Erlangen, Germany
}

\section{Abstract}

Normal and osteoarthritic human articular cartilage was investigated by in situ hybridization for expression patterns of the fibrillar collagens type I, II, and III to evaluate phenotypic changes of articular chondrocytes related to the disease. In 11 out of 20 samples, a defined subset of chondrocytes in the superficial and upper middle zone of osteoarthritic cartilage showed significant levels of cytoplasmic $\alpha 1$ (III) mRNA, whereas strong signals of $\alpha 1$ (II) mRNA were found in the upper and lower middle zone, partially overlapping with the zone of $\alpha 1$ (III) mRNA-expressing cells. The extent of type II and III collagen expression depended on the integrity of the extracellular matrix surrounding the chondrocytes, and the location within the articular cartilage. No $\alpha 1$ (I) mRNA was detectable in osteoarthritic original articular cartilage. The $\alpha 1$ (I) probe did, however, reveal signals in pannuslike tissue, osteophytes, and bone cells. In normal articular cartilage, no detectable levels of cytoplasmic mRNA for $\alpha 1$ (I), $\alpha 2$ (I), or $\alpha 1$ (III) were seen. Using specific mono- and polyclonal antibodies, we found deposition of type III collagen but hardly any of type I collagen in the superficial zone of osteoarthritic cartilage that is consistent with the in situ hybridization results.

These results indicate a phenotypic alteration in a defined subset of chondrocytes in conditions of diseased cartilage, expressing and synthesizing collagen type III independently from type I collagen, but in part simultaneously with type II collagen. ( J. Clin. Invest. 1993.91:829-837.) Key words: chondrocytes • in situ hybridization - collagen mRNA - articular cartilage • immunohistochemistry

\section{Introduction}

From studies on cartilage development in vitro and in situ, it has become evident that at least three distinct chondrocyte phenotypes can be distinguished on the basis of the collagen pattern expressed: ( $a$ ) chondrocyte precursor cells (e.g., limb bud or somite mesenchyme cells synthesizing type I collagen) (13 ); (b) differentiated hyaline chondrocytes (e.g., in embryonal cartilage anlagen, in the resting zone of fetal epiphyseal cartilage, or in articular cartilage expressing collagen types II, IX,

Please address correspondence to Dr. K. von der Mark, Max-PlanckSociety, Clinical Research Groups for Rheumatology at the Medical Clinic III, Schwabachanlage 10, 8520 Erlangen, Germany.

Received for publication 18 May 1992 and in revised form $28 \mathrm{Au}$ gust 1992

J. Clin. Invest.

(C) The American Society for Clinical Investigation, Inc

0021-9738/93/03/0829/09 \$2.00

Volume 91, March 1993, 829-837
$\mathrm{XI}$, and VI [for review see references 4-6]; and (c) hypertrophic chondrocytes in the growth zone of fetal epiphyseal or rib cartilage, synthesizing predominantly type $\mathrm{X}$ collagen (for review see reference 7). A fourth chondrocyte phenotype resembling the chondrocyte precursor cells can be induced in vitro by culturing and repeated passage of chondrocytes in monolayer, by BUdR, embryo extract, fibronectin, or retinoic acid (for review see reference 8 ). This modulation of the collagen expression pattern has been compared with a "dedifferentiation" and involves the transition to a fibroblastic cell shape, the formation of focal contacts and stress fibers, and synthesis of types I, III, and V collagen (8).

While extensive literature is available on the expression of these collagens by articular chondrocytes in vitro and in normal fetal and adult cartilage in vivo (9-11), relatively little is known on the collagen expression pattern by articular chondrocytes in degenerating cartilage. Several studies describe a stimulation of collagen synthesis as a response to cartilage degradation in experimentally induced osteoarthritic models ( 12 , 13 ) or in human osteoarthritis (14). The newly synthesized collagens seem to be predominantly type II collagen $(12,15)$ as well as type IX, XI, and VI collagen (16). There are, however, also reports on the identification of type I and III collagen in osteoarthritic cartilage by immunohistochemical and biochemical methods (17-19), indicating "dedifferentiation" of chondrocytes similar to the above described phenomenon in vitro. These results could not be confirmed by several other biochemical studies, which were not able to detect any type I collagen in osteoarthritic cartilage except in fibrocartilage $(14,15)$. In a recent study, areas of type $\mathrm{X}$ collagen deposition around chondrocyte clusters in osteoarthritic cartilage was shown indicating chondrocyte hypertrophy (20).

In this study, we have investigated the cartilage expression of collagen types I, II, and III in chondrocytes of normal and osteoarthritic human cartilage both at protein and mRNA level to check for phenotypic changes in vivo similar to those found in culture systems in vitro. We present evidence for the uncoupling of coordinated collagen expression pattern consisting either of types II, IX, and XI in differentiated, or of types I, III, and V in "dedifferentiated" chondrocytes, and describe subsets of chondrocytes expressing types II and III, but not type I collagen.

\section{Methods}

Tissue preparation and histochemistry. Histological sections were prepared from 20 mainly late stage osteoarthritic (knee arthroplasties/hip replacements) and eight normal cartilage specimens. In this study, only samples of original articular cartilage were investigated; osteophytes and other repair cartilage were excluded. Cartilage slices were fixed with $4 \%$ paraformaldehyde in PBS for $24 \mathrm{~h}$, dehydrated, embedded in paraffin, and 5-8- $\mu \mathrm{m}$ sections were cut. From all cartilage specimens, hematoxylin, toluidine blue, and safranin $\mathrm{O}$ staining were performed 
on parallel sections. The latter two were used to estimate the content of proteoglycans (21). All samples were histologically graded according to Mankin (22).

Immunohistochemistry. Deparaffinized sections were decalcified with 0.3 M EDTA ( $\mathrm{pH} 7.2$ ) and incubated with testicular hyaluronidase $(2 \mathrm{mg} / \mathrm{ml}, \mathrm{PBS}, \mathrm{pH} 5,30 \mathrm{~min}$ at room temperature). For the detection of type II collagen in normal and osteoarthritic samples, pretreatment with pronase $(1 \mathrm{mg} / \mathrm{ml}, \mathrm{PBS}, \mathrm{pH} 7.3,30 \mathrm{~min}$ at room temperature) was necessary. The staining pattern obtained with antibodies to type I collagen or $\mathrm{pN}-\alpha(\mathrm{III})$ was independent from section pretreatments with pronase. Primary antibodies were incubated for $1 \mathrm{~h}$ and visualized using alkaline-phosphatase-labeled secondary antibodies and 3-hydroxy-2-naphtylacid 2,4-dimethylanilid as color substrate. Nuclei were counterstained with hematoxylin. As negative control, the primary antibody was replaced either by preimmune serum or Trisbuffered saline ( $\mathrm{pH} 7.2)$.

A monoclonal antibody (CII D3) directed against an epitope in the CB 10 fragment of type II collagen was kindly provided by Dr. $R$. Holmdahl (University of Uppsala, Sweden) (23). Rabbit antisera against human type I collagen were purchased from Institut Pasteur (Lyon, France) or prepared and tested as described elsewhere (20). Polyclonal antibodies against type III procollagen were kindly provided by Dr. Günzler (Hoechst Co., Frankfurt, FRG). Preparation and specificity of this antibody are described in (24). Antibodies to the pN-propeptide of type III procollagen were shown to give identical staining patterns as antibodies to type III collagen since the majority of the pN-peptides remain attached to the type III collagen fibrils (25).

Preparation of RNA probes. For specific RNA probes, suitable fragments of human collagen chains $\alpha 1$ (I), $\alpha 2$ (I), $\alpha 1$ (II), and $\alpha 1$ (III) mRNA were selected and recloned into pGEM vectors (Promega Corp., Madison, WI) (for localization on the genes see Fig. 1). pHCG IN contains a StyI-KpnI fragment (207 bp) of the N-propeptide of collagen $\alpha 1$ (I) generated by the polymerase chain reaction technique using specific primers and total human fibroblast RNA. This fragment was cloned into HincII-KpnI sites of pGEM $4 Z$ and sequenced for control (Sequenase; United States Biochemical Corp., Cleveland, Ohio). pHCG $1 \mathrm{~A} 2$ contains a Xbal-MscI fragment (collagen $\alpha 2(\mathrm{I})$, $319 \mathrm{bp}$ ) from Hf 32 (kindly provided by Dr. Ramirez, Mt. Sinai School of Medicine, New York) (26), recloned in Xbal-HincII sites of pGEM 4Z. pHCG 2 contains a ECO RI-Dra I fragment (collagen $\alpha 1$ (II) 435 bp) from pHCAR 3 (kindly provided by Dr. Sandberg and Dr. Vuorio, University of Turku, Finland) (27) recloned in HincII-ECORI sites of pGEM 4Z. pHCG 3 contains a PstI-PstI fragment [collagen $\alpha 1$ (III], $294 \mathrm{bp}$ ) from pHFS3 (28) recloned in the Pst I site of pGEM 4Z; the required orientation (antisense transcripts using $\mathrm{T} 7$ polymerase) was checked by restriction analysis. pRNA 1 contains a Xbal-BamHI fragment (294 bp) of mouse 18S ribosomal RNA (rRNA) from pCM 1 (29) recloned in XbaI-BamI sites of pGEM 3Z. This probe shows $100 \%$ homology to human 18S rRNA and was used as a positive control.
The constructs were linearized and transcribed in vitro using $\mathrm{T} 7$ and SP6 RNA-polymerase (both from Promega) to generate antisense and sense transcripts respectively. The probes were labeled with $60-150$ $\mu \mathrm{Ci}\left(\alpha{ }^{35} \mathrm{~S}\right)$-UTP $(1,200 \mathrm{Ci} / \mathrm{mmol}$, New England Nuclear, Dreireich, FRG) per $0.5-1 \mu \mathrm{g}$ of template DNA to a specific activity of $\leq 1.2$ $\times 10^{9} \mathrm{cpm} / \mu \mathrm{g}$, and nonincorporated nucleotides were separated by alcohol precipitation. The quality of the transcripts was controlled using denaturating formaldehyde agarose gels. Northern blotting experiments using $5 \mu \mathrm{g}$ of total RNAs of human fetal chondrocytes, fibroblasts, and HT 1080 cells grown in monolayer cultures (F12 medium, $10 \%$ FCS) were performed to confirm the specificity of the transcripts and to avoid "sticky" probes. Total RNA was extracted using the $\mathbf{4} \mathrm{M}$ guanidine thiocyanate method (30).

In situ hybridization. In situ hybridization was performed as described in detail elsewhere (31). In brief, deparaffinized and rehydrated sections were digested with proteinase $\mathrm{K}(20 \mu \mathrm{g} / \mathrm{ml})$, postfixed, and acetylated in $0.25 \%$ acetic anhydride. The sections were hybridized for $12-16 \mathrm{~h}$ at $43^{\circ} \mathrm{C}$ with riboprobes at a final activity of $3-6 \times 10^{7} \mathrm{cpm} /$ $\mathrm{ml}$ depending on their length. After hybridization, the tissue sections were washed $40^{\circ} \mathrm{C} 2 \times \mathrm{SSC} / 0.5 \% \beta$-mercaptoethanol, and $40^{\circ} \mathrm{C} 0.5 \times$ SSC $/ 0.5 \% \beta$-mercaptoethanol, treated with RNAses A $(20 \mu \mathrm{g} / \mathrm{ml})$ and $\mathrm{T} 1(50 \mathrm{U} / \mathrm{ml})$, washed again for $2 \mathrm{~h} 45^{\circ} \mathrm{C} 2 \times \mathrm{SSC} / 50 \%$ formamide/ $0.5 \% \beta$-mercaptoethanol. After three times in $0.1 \times \mathrm{SSC}$ at room temperature, the slides were dehydrated.

Autoradiography was performed (Kodak NTB-2 nuclear track emulsion) for 3-4 d and sections were counterstained in $5 \%$ Giemsa dye.

\section{Results}

\section{Selection of RNA probes}

Because of high homologies existing between the closely related fibril-forming collagen genes, particular efforts were directed towards careful selection of DNA fragments to rule out crosshybridization effects (32). This is especially valid for in situ hybridization, where additional control informations such as transcript size, etc., are missing. Therefore, we selected and prepared collagen cDNA probes by the following criteria: $(a)$ Probes were selected for homology $<70 \%$ with other known collagen sequences available in the MIPSY-genbank (MaxPlanck-Institute for Biochemistry, Martinsried, FRG) using University of Wisconsin Genetics Computer Group programs (Fig. 2). (b) Northern blotting experiments using ${ }^{32} \mathrm{P}$-labeled antisense transcripts and total RNA extracted from human fetal chondrocytes [expressing $\alpha 1$ (II) mRNA], fibroblasts [expressing $\alpha 1$ (I), $\alpha 2$ (I), $\alpha 1$ (III) mRNA] showed specific bands

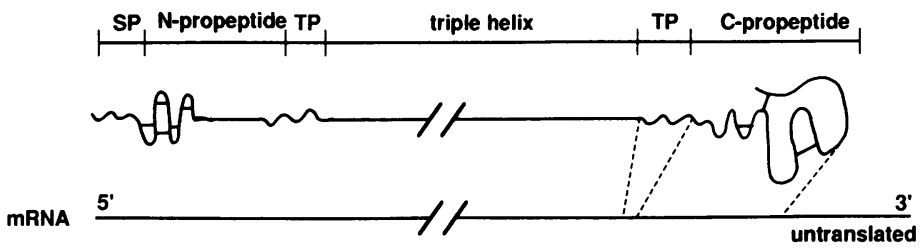

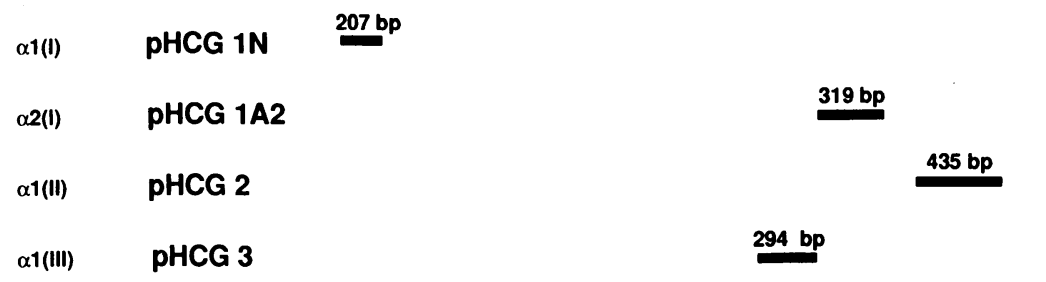

Figure 1. Diagram showing the position of the $\alpha 1$ (I), $\alpha 2$ (I), $\alpha 1$ (II), and $\alpha 1$ (III) cDNA probes in comparison to their corresponding mRNAs and different protein domains ( $S P$, signal peptide; $T P$, telopeptide). The length of the clones is indicated above the bars. 


\begin{tabular}{l|cccc} 
Collagens & $\alpha 1$ (I) & $\alpha 2($ I) & $\alpha 1$ (II) & $\alpha 1$ (III) \\
\hline Probes & & & & \\
\hline PHCG 1N & 100 & & $\left(73^{*}\right)$ & \\
\cline { 2 - 5 } PHCG 1A2 & 66 & 100 & 67 & 67 \\
\cline { 2 - 5 } PHCG 3 & 53 & & 100 & \\
\hline
\end{tabular}

Figure 2. Homologies in the nucleotide sequence between the collagen cDNA probes and other collagen DNA sequences. Only homologies $>50 \%$ are given. No significant homology could be found to nonfibril forming collagens or any other proteins. *Includes the additional second exon of collagen II (Ryan and Sandell, 1990 [41]).

with the respective probes at stringent washing conditions $\left(0.1 \times \mathrm{SSC}, 70^{\circ} \mathrm{C}, 0.1 \%\right.$ SDS $)$. Total RNA of HT 1080 cells (expressing neither of the collagen types I, II, or III) served as negative control. (c) Probes were tested for in situ hybridization in samples of human fetal epiphyseal cartilage (our own unpublished results). Osteoblasts (expressing only chains of type I collagen), endothelial cells of blood vessels (predominantly expressing type III collagen), and mesenchymal cells (expressing type I and III collagen ) served as internal specificity controls in the investigated specimens. RNA preservation and cell viability was controlled with the $18 \mathrm{~S}$ rRNA probe. Interestingly, the strength of the hybridization signals was less than expected from the anticipated abundance of the ribosomal RNA. This could be caused by secondary loop structures formed by the rRNAs, or caused by proteins of the ribosomal complex resistent to proteinase $\mathrm{K}$ digestion and thus preventing proper access of the probe. Sense RNA transcripts were used as negative control and showed only background signals in all samples.

\section{Independent expression of collagen type I, II, and III in osteoarthritic cartilage}

Upper and middle zone chondrocytes. In 15 out of 16 investigated samples of late stage osteoarthritic cartilage (Mankin grades 3-7) chondrocytes expressed considerable amounts of $\alpha 1$ (II) mRNA (Fig. $3 b$ ). These were confined to layers below the zone showing proteoglycan loss (Fig. 3, $b$ and $h$ ). An example representing middle to late phases of cartilage degeneration is depicted in Fig. 3, $a-h$, showing the surface fibrillated tibia plateau of a 70-yr-old patient (Mankin grade 4) with varus gonarthritis. The zone of chondrocytes with enhanced type II collagen expression is in 10 out of the 16 samples partially overlapping with a subset of chondrocytes showing significant expression of $\alpha 1$ (III) mRNA (Fig. $3 c$; for schematic representation, see Fig. 4). In none of the investigated samples was expression of $\alpha 1$ (III) mRNA found without simultaneous expression of $\alpha 1$ (II) mRNA in the same or adjacent cells. Lower middle zone chondrocytes showed mostly only $\alpha 1$ (II) mRNA, with very few cells also positive for $\alpha$ l (III) in areas of extensive proteoglycan depletion. The intensity of the signals obtained with the $\alpha 1$ (III) probe in chondrocytes were comparable or lower than that of $\alpha 1$ (II) mRNA present in the same specimens. Overall, more chondrocytes expressed $\alpha 1$ (II) than $\alpha 1$ (III) mRNA (Fig. 3, $b$ and $c$ ). Although direct evidence for coordinate expression of type II and III collagens in the same cells would require double-labeling techniques, comparison of consecutive sections provided conclusive evidence for the simultaneous expression of both collagen genes in some areas (Fig. $3 b$ and $c ; 5 g$ and $h$ ). In none of the investigated samples $\alpha 1$ (I) or $\alpha 2$ (I) mRNA expression was seen in upper and middle zone chondrocytes (Fig. 3, $a$ and $i$ ), in contrast to fibroblastic cells in pannuslike tissue overlaying the articular cartilage in an osteoarthritic femur condyle of a 65 -yr-old patient who showed signals with the $\alpha 1$ (I) and $\alpha 2$ (I) probes (Fig. $3 i$ ). Pannuslike tissue also showed cytoplasmic $\alpha 1$ (III) mRNA (Fig. $3 k$ ). Chondrocytes of the superficial zone were negative for both $\alpha 1$ (II) and $\alpha 1$ (III) in sites of severe depletion of the matrix of proteoglycans. These cells were, however, in all cases viable as shown by in situ hybridization with the 18S rRNA probe (Fig. 3, $d$ and $l$ ).

\section{Chondrocytes of the deep zone and calcified cartilage}

Chondrocytes of the deep zone of osteoarthritic original cartilage expressed no $\alpha 1$ (III), $\alpha 1$ (I) or $\alpha 2$ (I) collagen mRNAs. The only exception was found in one sample of an osteoarthritic femoral head ( $79 \mathrm{yr}$, female), in which deep zone chondrocyte clusters showed simultaneous expression of $\alpha 1$ (II) and $\alpha 1$ (III), and remarkable signals with the $\alpha 2$ (I) probe (Fig. 5 $f$ ), but not with the $\alpha 1$ (I) probe (Fig. $5 a$ ). This area was localized in the immediate neighborhood to a new-forming secondary cartilage. This finding of cytoplasmic $\alpha 2$ (I) mRNA in the absence of $\alpha 1$ (I) mRNA was unexpected in view of the fact that $[\alpha 1(\mathrm{I})]_{3}$ molecules, but not $[\alpha 2(\mathrm{I})]_{3}$ molecules are thermodynamically stable (33). Type II collagen expressing cells were regularly found in the deep zone, but not in the lower part and in the calcified zone (see also reference 31). Strong $\alpha 1$ (I) and $\alpha 2(\mathrm{I})$ expression was usually found in osteoblastic cells lining the subchondral bone trabeculae.

\section{Chondrocytes in normal human articular cartilage and early stages of cartilage degeneration}

Chondrocytes in normal adult articular cartilage did not show expression of $\alpha 1(\mathrm{I}), \alpha 2(\mathrm{I})$, and $\alpha 1$ (III) mRNA in any zone (Fig. $5 a, b$, and $d$ ) and only exceptionally weak signals for $\alpha 1$ (II) mRNA (see also reference 31). This is consistent with the low metabolic activity reported for adult healthy articular chondrocytes in situ. In samples of earlier stages of cartilage degeneration (Mankin grades 2-4, obtained from autopsied patients not clinically diagnosed as osteoarthritis) $\alpha 1$ (II) mRNA, but only few $\alpha 1$ (III) expressing chondrocytes were found (one out of four cases, not shown). Again no cytoplasmic $\alpha 1$ (I) or $\alpha 2$ (I) mRNA was seen in chondrocytes.

\section{Deposition of type III collagen in the extracellular matrix}

To confirm the collagen expression patterns found by in situ hybridization and to investigate whether the identified mRNAs are translated into collagen, parallel sections were stained using collagen type I-, II-, and III-specific mono- and polyclonal antibodies. The extracellular matrix of normal cartilage was positive for type II collagen. Type I and III collagen were only seen in a very thin layer at the surface, showing reduced type II and proteoglycan staining (not shown, see also (31)). This layer may be developmentally derived from the fetal epichondral zone, which shows a similar composition $(9,11$, and our own unpublished results). In contrast, the extracellular matrix of osteoarthritic cartilage showed significant pericellular and interterritorial deposition of type III collagen of various degrees. 

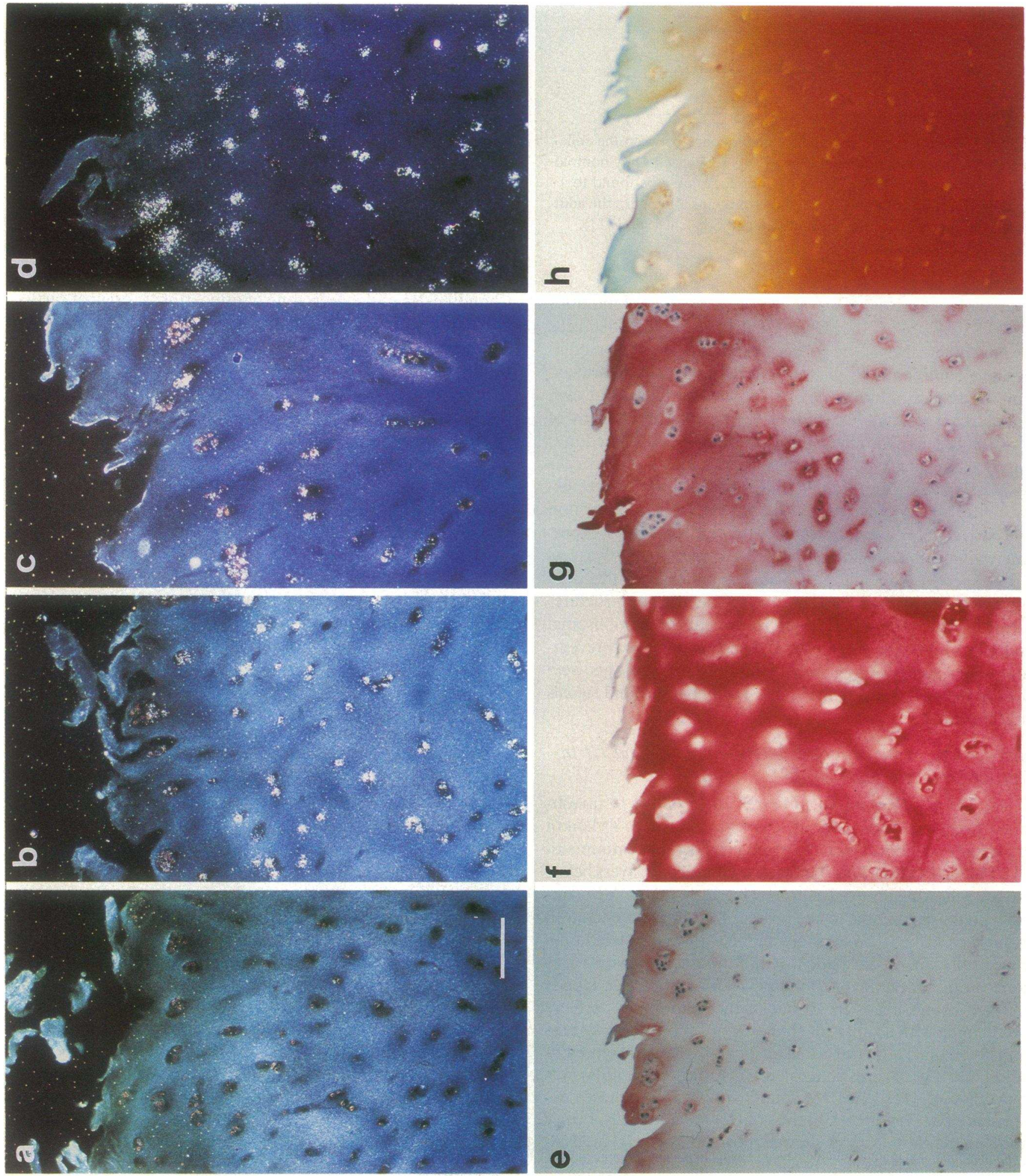

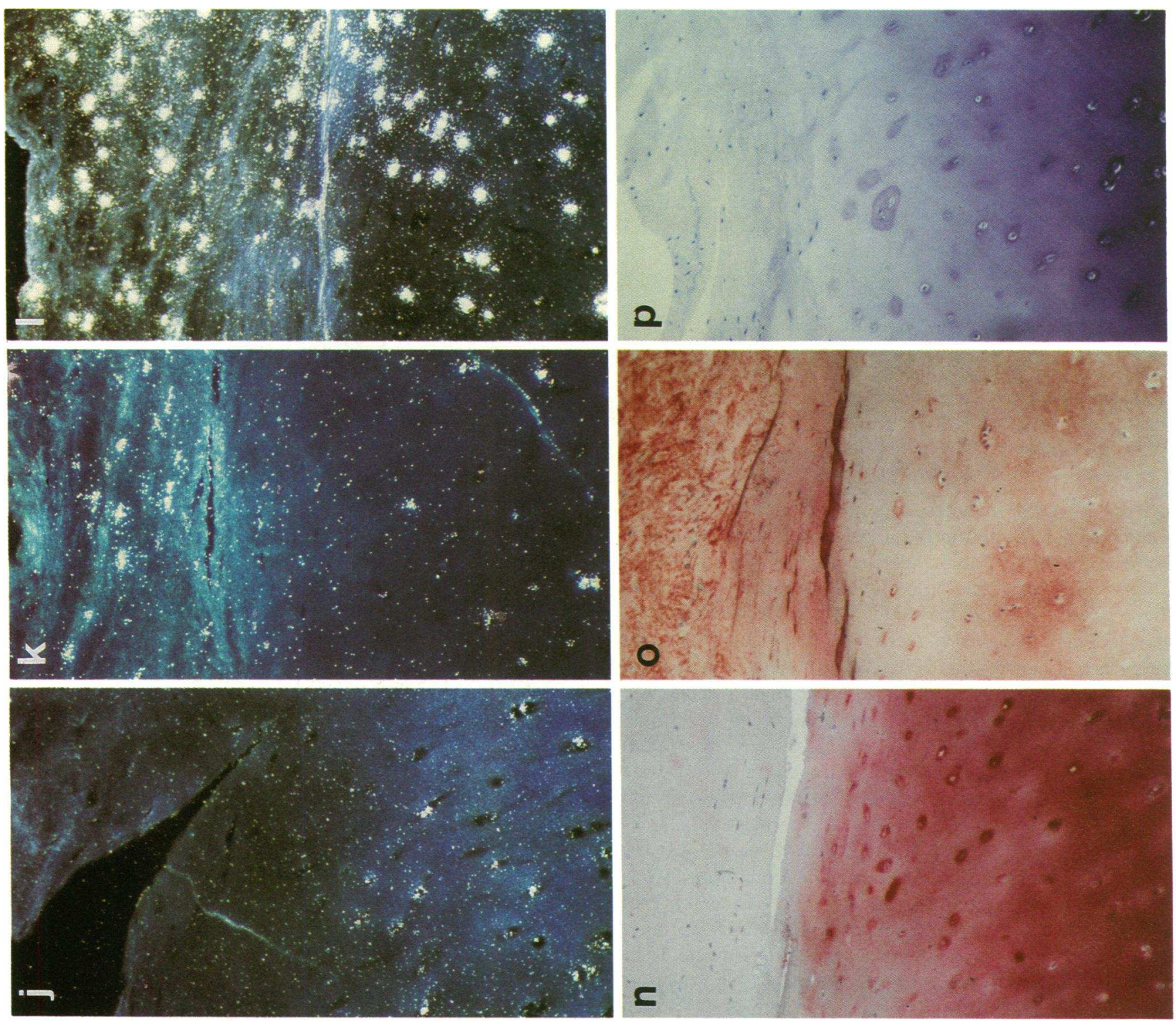

$\infty$
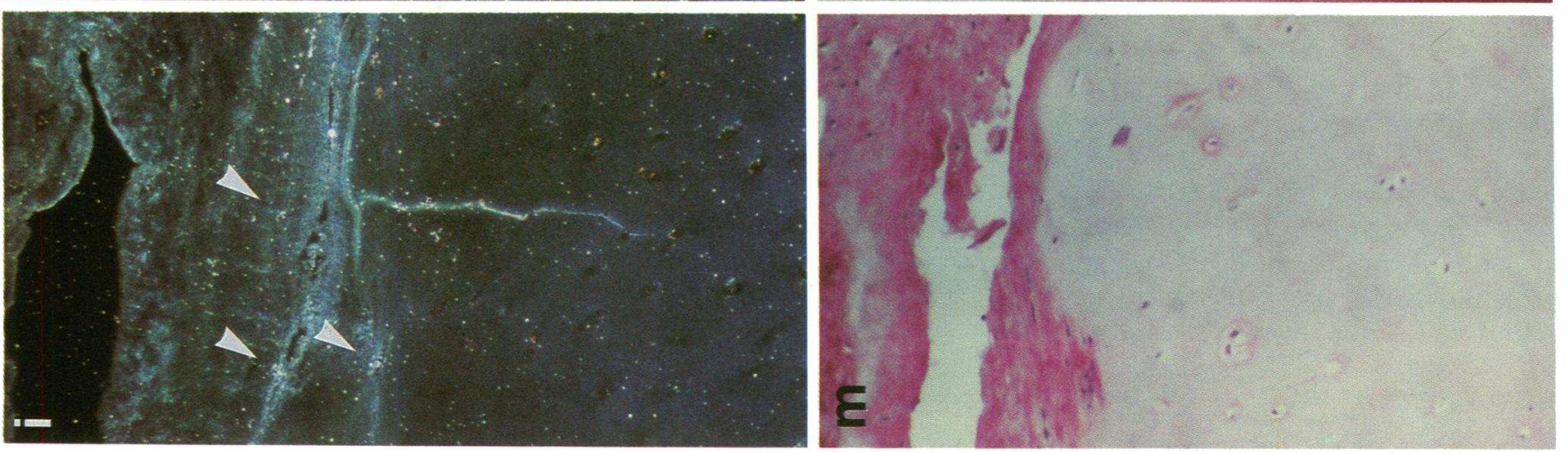


\section{chondrocytes}

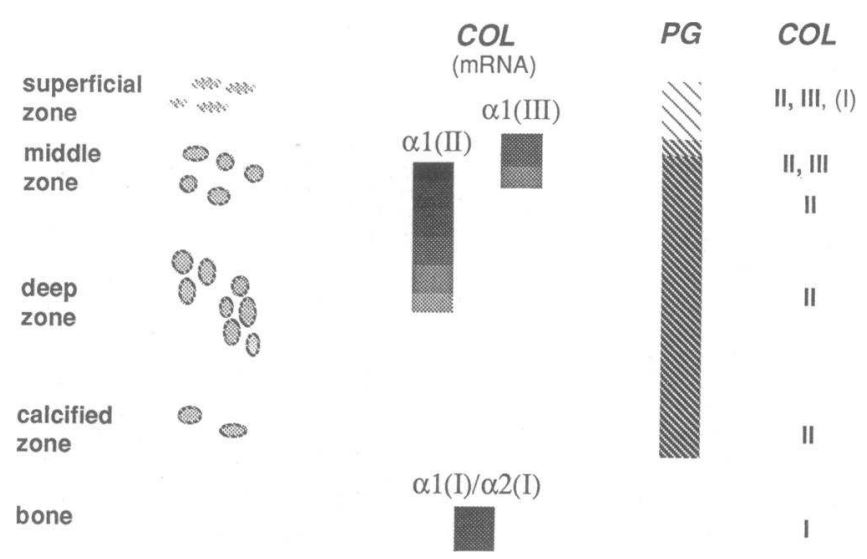

Figure 4. Schematic presentation of a typical collagen expression pattern seen in late stage osteoarthritic cartilage (Mankin grade 3-7) in correlation to the proteoglycan staining. In 6 of 11 cases, however, the zone of type III collagen expressing cells did not clearly extent beyond the zone of type II collagen expressing cells. $I$, weak staining with the anti-human type I antibodies.

Fig. $3 g$ shows a case of intense deposition of type III collagen in the superficial zone and in the upper middle zone of surface fibrillated osteoarthritic tibia plateau (same sample as shown in Fig. 3, $a-d$ ). The distribution is consistent with the pattern of type III mRNA expression (Fig. 3, $c$ and $k$ ). Nevertheless, type II collagen remained the most prominent collagen in the interterritorial matrix (Fig. $3 f$ ), although areas of local periand intercellular type II collagen losses were also frequently observed (see also references 20,31 ). In accordance with the mRNA hybridization results, less type III collagen was found in early stage specimens. Furthermore, collagen type III was detected together with type I in fibrous pannuslike tissue layers overlaying or infiltrating arthritic articular cartilage (Fig. 3, m and $o$ ). In some samples of osteoarthritic cartilage, staining for type I collagen was found at the surface and around superficial and middle zone chondrocytes (Fig. $3 e$ ), but considerably less than type III collagen. Interestingly, no type I collagen staining was obtained in or around chondrocyte clusters in the deep zone of one osteoarthritic cartilage sample that had shown positive signals with the probe $\alpha 2$ (I), but not with the $\alpha 1$ (I) probe (Fig. 5, $e$ and $f$ ).

\section{Discussion}

In this study, we provide evidence for significant alterations in the collagen expression pattern by articular chondrocytes in osteoarthritis. In the superficial and upper middle layers of osteoarthritic cartilage (Mankin grades 3-7), chondrocyte clusters and single chondrocytes were identified expressing type III collagen besides type II collagen, but not type I collagen. This zone of type III collagen expressing cells was often surface-fibrillated and partially correlated with the surface zone of reduced proteoglycan staining. It was partially overlapping with the zone of chondrocytes showing enhanced type II collagen expression (see Fig. 4 and reference 31 ). The expression of cytoplasmic $\alpha 1$ (III) mRNA identified by in situ hybridization was consistent with the immunohistochemical localization of extracellular type III collagen in this area. By antibody staining, however, also some extracellular type I collagen was identified in the upper zone; the corresponding cytoplasmic $\alpha 1(\mathrm{I})$ and $\alpha 2$ (I) mRNA levels may have been too low to be detected by in situ hybridization.

The extent of staining and the number of cells showing $\alpha 1$ (III) mRNA signals varied considerably not only among samples but also within the samples. The variety of collagen alterations in osteoarthritic cartilage is also documented by the identification of type $\mathrm{X}$ collagen deposition around clusters of chondrocytes in the upper, fibrillated zone (20) similar to that shown in this study. Double immunostaining experiments will be required to elucidate the relative distribution of type $\mathrm{X}$ and III collagens in osteoarthritic cartilage.

The heterogeneity in cartilage alterations and sampling problems may explain conflicting reports on the biochemical analysis of osteoarthritic cartilage, showing the predominance of type II collagen $(15,16)$ or the presence (17-19) or absence $(12,14,15)$ of significant amounts of type I and III collagen. The latter collagens will appear in cartilage extracts when overlaying or infiltrating, macroscopically hardly visible fibrous tissue is not excluded (15). Our studies clearly indicate that a large number of chondrocytes in the original osteoarthritic articular cartilage of late stages (Mankin grades 3-7) express type II collagen, and that the major collagen visible by antibody staining is type II collagen, in particular in the middle and deep zone (see also references 20,31 ), thus confirming the results by Goldwasser et al. (15), Eyre et al. (12), and Ronzière et al. (16). In the fibrillated surface layer, however, type III collagen seems to be a significant constituent in certain areas of the cartilage matrix and may thus have an impact on the stability of the collagen matrix in this zone. At the moment, however, no quantitative data on the relative amounts of type II and type III collagens in this zone are available.

Our results can not confirm the synthesis or presence of major amounts of collagen type I outside of overlaying fibrous tissue. This demonstrates the value of the local resolution of immunohistochemical and in situ hybridization methods supplementing biochemical examinations.

The lack of expression of type I collagen, at least below the detection level, besides high levels of type II and III collagen or type III alone in osteoarthritic chondrocytes is unexpected and not in accordance with the current view of discrete chondrocyte phenotypes defined by a coordinate regulation of either type II, IX, and XI collagens as characteristic for functional

Figure 3. In situ hybridization analysis of collagen expression $(a-d$ and $i-l)$ and histochemical staining of collagens and proteoglycans $(e-h$ and $m-p)$ in sections of human osteoarthritic cartilage. For detection of cytoplasmic mRNA, cartilage sections were hybridized with ${ }^{35}$ S-RNA probes specific for $\alpha 1$ (I) ( $a) ; \alpha 2(\mathrm{I})(i) ; \alpha 1$ (II) ( $b$ and $j) ; \alpha 1$ (III) ( $c$ and $k$ ); and 18S rRNA ( $d$ and $l)$. Extracellular collagens were immunostained with a rabbit anti human type I collagen antibody $(e$ and $m)$, a monoclonal anti-type II collagen antibody $(f$ and $n)$, and a rabbit anti-PN $\alpha 1$ (III) antibody ( $g$ and $o$ ), followed by alkaline phosphatase labeled second antibodies. $(h)$ safranin $\mathrm{O},(p)$ toluidine blue staining, both for proteoglycans. $(a-h)$ Varus gonarthritis, tibia plateau ( $70 \mathrm{yr}$, female) Mankin grade 4; $(i-p)$ Osteoarthritic femur condyle (65 yr, female) Mankin grade 5. Bar, $100 \mu \mathrm{m}$. 

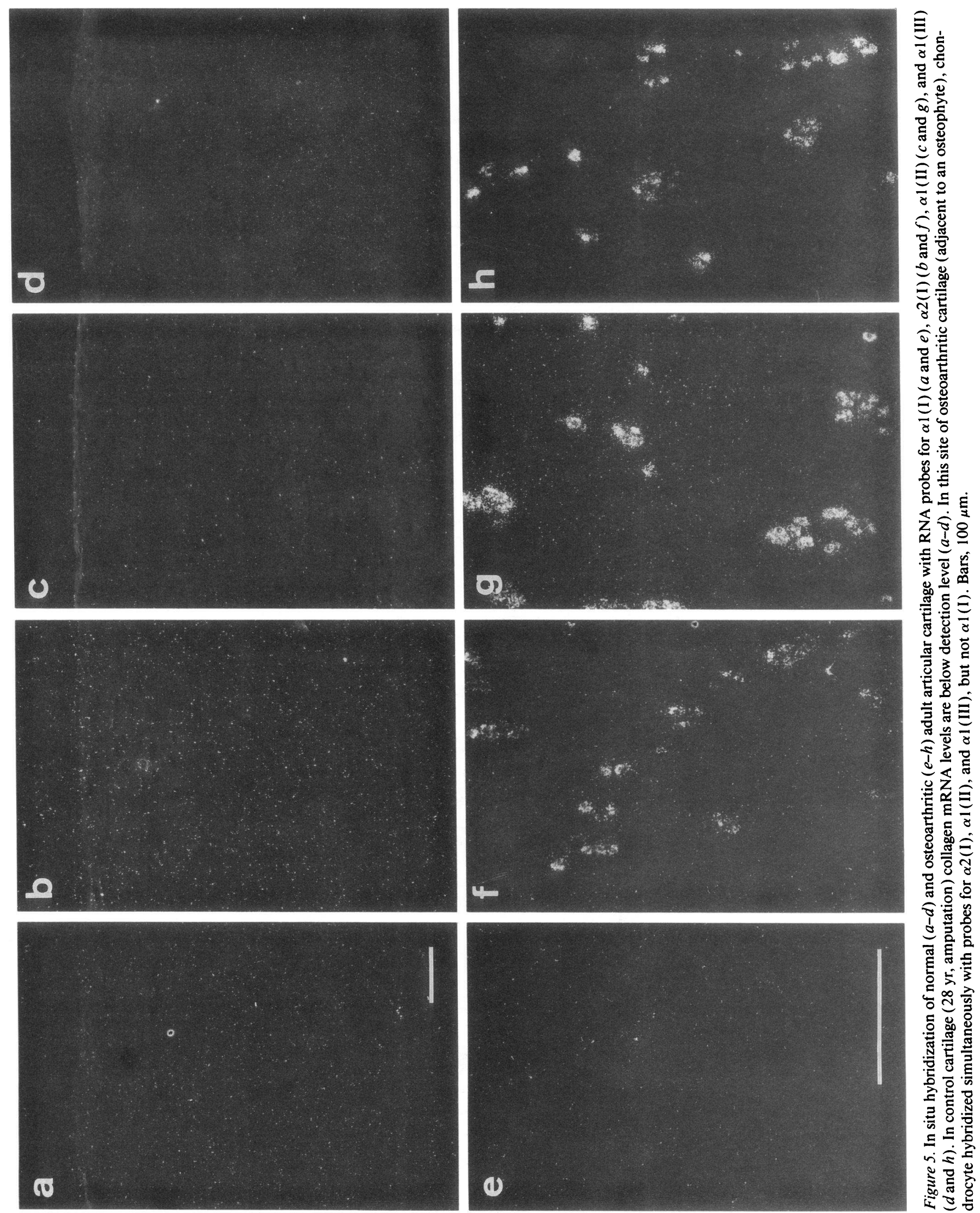
resting chondrocytes, or of types I, III, and V collagens described for the "dedifferentiated" or modulated phenotype in vitro $(8,10,34,35)$. In noncartilagenous tissue, collagen type III is expressed in vivo and in vitro mostly together with collagen type I, though in different ratios $(36,37)$. In chondrocyte cultures modulated in vitro, a rather abrupt switch from type II to types I and III collagen synthesis is observed with type I as the most abundant protein synthesized (10, 35, 38), suggesting that both collagens are coregulated. The fact that we cannot detect type I collagen mRNA in osteoarthritic chondrocytes demonstrates that the phenotypic changes of osteoarthritic chondrocytes described in this study is different from the dedifferentiation event of chondrocytes seen in vitro. Our results are in favor of an uncoupled, individual regulation of single collagen genes in the chondrocyte in situ.

This seems also the case for the COL1A1 and COL1A2 genes. In one sample, we found signals with the $\alpha 2$ (I) probe in chondrocytes of the deep zone of osteoarthritic cartilage in the absence of $\alpha 1$ (I) transcripts, while the same $\alpha 1$ (I) probe revealed strong signals in osteoblasts in the same sample. Since no type I collagen staining could be achieved in the environment of these chondrocytes using a polyclonal antibody, we assume that these " $\alpha 2$ (I)" transcripts may represent the noncollagenous, cartilage-specific transcript of the COL1A2 gene, controlled by a different promotor (39). To distinguish between both alternative transcripts, probes derived from the differing 5'-ends will be necessary.

The factors and mechanisms regulating transcription and translation of collagen types in osteoarthritic cartilage are not yet known. Systemic factors like cytokines produced (e.g., from synovial tissue) may affect the collagen expression pattern. For example, interleukin 1 enhances type I and III collagen in human chondrocytes and suppresses the expression of the cartilage-specific collagens in vitro (40). However, the fact that the alterations in collagen expression pattern in osteoarthritis are not evenly distributed over the articular cartilage, but different in marginal or weight bearing areas, suggest an additional strong impact by the local environment; e.g., the integrity of the extracellular matrix and mechanical factors.

In conclusion, we were able to demonstrate expression and synthesis of collagen type III by arthritic articular chondrocytes and its deposition in the extracellular matrix of articular cartilage. The finding of chondrocytes expressing $\alpha 1$ (III) mRNA either solely or together with $\alpha 1$ (II), both in the absence of $\alpha 1$ (I) and $\alpha 2$ (I) collagen mRNAs, displays an in vivo phenotype of articular human chondrocytes different to those described so far in vivo and in vitro. This presumably implicates different regulatory mechanisms responsible for the phenotypic changes seen in articular chondrocytes in vivo. Furthermore, this study shows a considerable heterogeneity of collagen alterations and phenotypic changes of chondrocytes among and within various samples of arthritic cartilage that can be identified by a combination of immunohistological and in situ hybridization methods. Further systematic studies on a larger number of samples and careful correlation to clinical and pathological features of the arthritic cartilage samples may allow conclusions on the sequence of phenotypic alterations articular chondrocytes undergo during cartilage degeneration.

\section{Acknowledgments}

We thank Ms. Marion van Menxel and Maria Schmidt for expert technical assistance, and T. Kirsch, Max-Planck-Gesellschaft (Erlangen), and Dr. V. Günzler, Hoechst Co. (Frankfurt), for generously providing us with antibodies to human type I collagen, and PN- $\alpha 1$ (III). This work was supported by the Bundesministerium für Forschung und Technik (BMFT grant 01VM8702/0).

\section{References}

1. Linsenmayer, T. F., B. P. Toole, and R. L. Trelstad. 1973. Temporal and spatial transations in collagen types during embryonic chick limb development. Dev. Biol. 35:232-239.

2. von der Mark, K., H. von der Mark, and S. W. Gay. 1976. Study of differential collagen synthesis during development of the chick embryo by immunofluorescence. Dev. Biol. 53:153-170.

3. von der Mark, K., and H. von der Mark. 1977. Immunological and biochemical studies of collagen type transition during in vitro chondrogenesis of chick limb mesodermal cells. J. Cell Biol. 73:736-747.

4. Mayne, R., and K. von der Mark. 1983. Collagens of cartilage. In Cartilage. B. K. Hall, editor. Academic Press, New York. 181-214.

5. Mayne, R. 1989. Cartilage collagens. What is their function, and are they involved in articular disease? Arthritis Rheum. 32:241-246.

6. Eyre, D. R., J.-J. Wu, and P. E. Woods. 1991. The cartilage collagens: structural and metabolic studies. J. Rheumatol. 18 (Suppl. 27):49-51.

7. Schmid, T. M., R. G. Popp, and T. F. Linsenmayer. 1990. Hypertrophic cartilage matrix. Ann. NY Acad. Sci. 580:64-73.

8. von der Mark, K. 1986. Differentiation, modulation and dedifferentiation of chondrocytes. Rheumatology. 10:272-315.

9. von der Mark, K., and H. Von der Mark. 1977. The role of the three genetically distinct collagen types in endochondral ossification and calcification of cartilage. J. Bone J. Surg. Br. Vol. 59-B-4:458-464.

10. Benya, P. D., S. R. Padilla, and M. E. Nimni. 1978. Independent regulation of collagen types by chondrocytes during the loss of differentiated function in culture. Cell. 15:1313-1321.

11. Sandberg, M., and E. Vuorio. 1987. Localization of types I, II, and III collagen mRNAs in developing human skeletal tissues by in situ hybridization. $J$. Cell Biol. 104:1077-1084.

12. Eyre, D. R., C. A. McDevitt, M. E. J. Billingham, and H. Muir. 1980. Biosynthesis of collagen and other matrix proteins by articular cartilage in experimental osteoarthrosis. Biochem. J. 188:823-837.

13. Floman, Y., D. R. Eyre, and M. Glimcher. 1980. Induction of osteoarthrosis in the rabbit knee joint: biochemical study on the articular cartilage. Clinical Orthopaedics and Related Research 147:278-286.

14. Lippiello, L., D. Hall, and H. J. Mankin. 1977. Collagen synthesis in normal and osteoarthritic human cartilage. J. Clin. Invest. 59:593-600.

15. Goldwasser, M., T. Astley, M. Van der Rest, and F. H. Glorieux. 1982. Analysis of the type of collagen present in osteoarthritic human cartilage. Clin. Orthop. Relat. Res. 167:296-302.

16. Ronzière, M.-C., S. Ricard-Blum, J. Tiollier, D. J. Hartmann, R. Garrone, and D. Herbage. 1990. Comparative analysis of collagens solubilized from human fetal, and normal and osteoarthritic adult articular cartilage, with emphasis on type VI collagen. Biochim. Biophys. Acta. 1038:222-230.

17. Nimni, M. E., and K. Deshmukh. 1973. Differences in collagen metabolism between normal and osteoarthritic human articular cartilage. Science (Wash. DC). 181:751-752.

18. Adam, M., and Z. Deyl. 1983. Altered expression of collagen phenotype in osteoarthrosis. Clin. Chim. Acta. 133:25-32.

19. Gay, S. W., P. K. Müller, C. Lemmen, K. Remberger, K. Matzen, and K. Kühn. 1976. Immunohistological study on collagen in cartilage-bone metamorphosis and degenerative osteoarthrosis. Klin. Wochenschrift. 54:969-976.

20. von der Mark, K., T. Kirsch, A. G. Nerlich, A. Kuss, G. Weseloh, K. Glückert, and H. Stöss. 1992. Type X collagen synthesis in human osteoarthritic cartilage: Indication of chondrocyte hypertrophy. Arthritis Rheum. 35:806-811.

21. Rosenberg, L. 1971. Chemical basis for the histological use of safranin O in the study of articular cartilage. J. Bone Jt. Surg. Am. Vol. 53-A:69-82.

22. Mankin, H. J., H. Dorfman, L. Lippiello, and A. Zarins. 1971. Biochemical and metabolic abnormalities in articular cartilage from osteo-arthritic human hips. J. Bone Jt. Surg. Am. Vol. 53-A:523-537.

23. Holmdahl, R., K. Rubin, L. Klareskog, E. Larsson, and H. Wigzell. 1986. Characterization of the antibody response in mice with type II collagen-induced arthritis, using monoclonal anti-type II collagen antibodies. Arthritis Rheum. 29:400-416.

24. Nowack, H., S. Gay, G. Wick, U. Becker, and R. Timpl. 1976. Preparation and use in immunohistology of antibodies specific for type 1 and type III collagen and procollagen. J. Immunol. Methods. 12:117-124.

25. Fleischmajer, R., R. Timpl, L. Tuderman, L. Raisher, M. Wiestner, J. S. Perlish, and P. N. Graves. 1981. Ultrastructural identification of extension aminopropeptides of type I and type III collagens in human skin. Proc. Natl. Acad. Sci. USA. 78:7360-7364.

26. Bernard, M. P., J. C. Myers, M.-L. Chu, F. Ramirez, E. F. Eikenberry, and D. J. Prockop. 1983. Structure of a cDNA for the pro $\alpha 2$ chain of human type I 
procollagen. Comparison with chick cDNA for $\alpha 2$ (I) identifies structurally conserved features of the protein and the gene. Biochemistry. 22:1139-1145.

27. Elima, K., T. Vuorio, and E. Vuorio. 1987. Determination of the single polyadenylation site of the human pro $\alpha$ ( II) collagen gene. NAR 15-22:94999504.

28. Sandberg, M., J. K. Mäkelä, P. Multimäki, T. Vuorio, and E. Vuorio. 1989. Construction of a human pro $\alpha$ I (III) collagen cDNA clone and localization of type III collagen expression in human fetal tissues. Matrix. 9:82-91.

29. Grummt, I., C. Soellner, and I. Scholz. 1979. Characterization of a cloned ribosomal fragment from mouse which contains the $18 \mathrm{~S}$ coding region and adjacent spacer sequences. NAR 6:1351-1369.

30. Sambrook, J., E. F. Fritsch, and T. Maniatis. 1989. Molecular cloning-A laboratory manual. Cold Spring Harbor Laboratory Press.

31. Aigner, T., H. Stöss, G. Weseloh, G. Zeiler, and K. von der Mark. 1992. Activation of collagen type II expression in osteoarthritic and rheumatoid cartilage. Virchows Arch. B Cell Pathol. 62:337-345.

32. Hayashi, M., Y. Ninomiya, J. N. Parsons, K. Hayashi, B. R. Olsen, and R. L. Trelstad. 1986. Differential localization of mRNAs of collagen types I and II in chick fibroblasts, chondrocytes, and corneal cells by in situ hybridization using cDNA probes. J. Cell Biol. 102:2302-2309.

33. Tkocz, C., and K. Kühn. 1969. The formation of triple-helical collagen molecules from alpha-1 or alpha-2 polypeptide chains. Eur. J. Biochem. 7:454462.

34. Mayne, R., M. S. Vail, P. M. Mayne, and E. J. Miller. 1976. Changes in type of collagen synthesized as clones of chick chondrocytes grow and eventually lose division capacity. Proc. Natl. Acad. Sci. USA. 73:1674-1678.

35. von der Mark, K., V. Gauss, H. Von der Mark, and P. K. Müller. 1977. Relationship between cell shape and type of collagen synthesised as chondrocytes lose their cartilage phenotype in culture. Nature (Lond.). 267:531-532.

36. Hill, R. J., and E. Harper. 1984. Quantitation of types I and III collagens in human tissue samples and cell culture by cyanogen bromide peptide analysis. Anal. Biochem. 141:83-93.

37. Layman, D., E. Epstein, R. Dodson, and J. Titus. 1977. Biosynthesis of type I and III collagens by cultured smooth muscle cells from human aorta. Proc. Natl. Acad. Sci. 74:671-675.

38. Mayne, R., B. W. Elrod, P. M. Mayne, R. D. Sanderson, and T. F. Linsenmayer. 1984. Changes in the synthesis of minor cartilage collagens after growth of chick chondrocytes in 5-bromo-2'-deoxyuridine or to senescence. Exp. Cell Res. 151:171-182.

39. Bennett, V. D., I. M. Weiss, and S. L. Adams. 1989. Cartilage-specific $5^{\prime}$ end of chick $\alpha 2$ (I) collagen mRNAs. J. Biol. Chem. 264:8402-8409.

40. Goldring, M. B., J. R. Birkhead, L. J. Sandell, T. Kimura, and S. M. Krane. 1988. Interleukin 1 suppresses expression of cartilage-specific types II and IX collagens and increases types I and III collagens in human chondrocytes. $J$. Clin. Invest. 82:2026-2037.

41. Ryan, M. C., and L. J. Sandell. 1990. Differential expression of a cysteinerich domain in the amino-terminal propeptide of type II (cartilage) procollagen by alternative splicing of messenger RNA. J. Biol. Chem. 265:10336-10339. 